\title{
Inmersión educativa e interaprendizaje en equipo
}

\section{Educational immersion and team inter-learning}

\author{
Julián Alberto Berrocal Camarena*I \\ *Departamento Académico de Educación, Facultad de Educación, Universidad Nacional del Centro del Perú (UNCP), Huancayo-Perú.
}

\begin{abstract}
Resumen
Este artículo se centra en una investigación cualitativa etnoeducativa. Plantea como se va estableciendo la conformación del equipo, los cronogramas de las reuniones de trabajo, el respeto por las normas de convivencia, el trabajo de interaprendizaje, el desarrollo de las actividades de aprendizaje y las autoevaluaciones. Para describir las experiencias de interaprendizaje del equipo de Regalado en la Institución Educativa Ángela Moreno de Gálvez de Tarma.
\end{abstract}

Palabras Claves: Inmersión educativa, aprendizaje, interaprendizaje, trabajo de equipo, sesión de aprendizaje.

\begin{abstract}
This article focuses on qualitative ethno-educational research. It outlines how the formation of the team is established, the schedules of the work meetings, respect for the rules of coexistence, inter-learning work, the development of learning activities and self-evaluations. To describe the inter-learning experiences of the Regalado team at the Angela Moreno de Galvez de Tarma Educational Institution.
\end{abstract}

Keywords: Educational immersion, learning, inter-learning, teamwork, learning session.

1 Correspondiencia: Julián Alberto Berrocal Camarena, albertoberrocal777@gmail.com 


\section{Imersão educativa e interaprendizagem em equipe}

\section{Resumo}

Neste artigo se centraliza em uma investigação qualitativa Etna educativa. Planeja como se vai estabelecendo a conformação da equipe, os cronogramas das reuniões de trabalho, o respeito pelas normas de convivência, o trabalho de Inter aprendizagem, o desenvolvimento das atividades de aprendizagem e a auto avaliação. Para descrever as experiências de Inter aprendizagem da equipe do Sr. Regalado na Instituição Educativa Ângela Moreno de Gálvez de Tarma.

Palavras Claves: Imersão educativa, aprendizagem, Inter aprendizagem, trabalho de equipo, sessão de aprendizagem.

\section{Llamkay educativam uñunakuna yachaspanchik}

\section{Uchuycha}

Kayarticulumqawan investigación cualitativa ednoeducativanisqan. Chaypin ninuñaskarurakuna, imaynasllamkasun, uñanakuspa, sumaqyachaywan, llamkaspayachaykunata, ruwaspamakiruraymantayachayqawaspa imaynaruwasqanchikta. Ruwanapaqtiktuqayyachaymantallapallanquchumanta Chay regaladoInstitución Educativa Ángela Moreno de Gálvez Tarmamanta.

Hatun apup simi: Llamkay educativam, yachay, yachaspanchik, llamkay uñu, sesión chay yachay. 


\section{Introducción}

En estos últimos tiempos se está fortaleciendo la investigación cualitativa, especialmente la investigación etnoeducativa. Establece los tiempos para las reuniones del equipo, desde una perspectiva de solidaridad y dedicación, la elaboración del diseño establecido por el equipo de Regalado. Con la finalidad de describir la experiencia de interaprendizaje en el marco de la inmersión educativa.

Así mismo se considera la conceptualización, el registro, codificación y análisis de datos, validez y confiabilidad y la selección de investigadores endógenos. Como parte de las categorías de la investigación etnoeducativo. Para describir la misma experiencia de interaprendizaje en equipo y cómo se va estructurando y conformando en el aula, para lograr los objetivos y metas de los aprendizajes.

De todos modos, es necesario preguntarse ¿Cómo las estudiantes del equipo de Regalado perciben el interaprendizaje en equipo? Considerando el principio que todas sus compañeras son parte del equipo, asumen compromisos en el proceso de planificación y desarrollo de las actividades de aprendizaje en el aula. Para realizar los trabajos según los planificado antes de cada sesión de aprendizaje.

\section{Inmersión educativa}

\section{Concepto}

Es un método que corresponde a la investigación cualitativa etnoeducativa. Radica en establecer el tiempo para las reuniones: solidaridad y dedicación, el diseño del proyecto por el equipo, la conceptualización, el registro, codificación y análisis de datos, validez y confiabilidad, así como la selección de investigadores endógenos. Con la finalidad de conocer la realidad de las estudiantes del equipo de Regalado de la sección E, en su trabajo en equipo de interaprendizaje, como parte de la inmersión educativa, a través de la investigación realizada por alguno de sus miembros.

Así mismo el equipo de Regalado entiende que todas deben incluirse en el interaprendizaje, a través de videos, Facebook y debates. Este entendimiento muestra una concepción de cooperación e inclusión, porque conciben esta forma de aprendizaje como la mejor forma de movilizar las competencias en las diferencias áreas. Por ello consideran a sus compañeras como parte fundamental del desarrollo personal y del equipo para los mayores logros y beneficios (Olivares Olivares, y otros, 2018, pág. 94).

\section{Categorías}

El tiempo establecido para las reuniones con el equipo de Regalado fue de: 90 minutos en cada clase. Se tuvo la experiencia de interaprendizaje etnoeducativa, en el cual se logra el intercambio de experiencias, vivencias, ideas, que han establecido en su práctica del aprendizaje. Para determinar las tareas y funciones, para que todo marche bien y sobre ruedas, en el logro de sus competencias.

En estas reuniones de interaprendizaje el equipo de Regalado establece las normas de convivencia. Como parte del compromiso que asumen las integrantes del equipo en el proceso y desarrollo de planificación del aprendizaje. Para fortalecer las capacidades y habilidades, porque cada integrante es importante, por lo tanto, nadie debe quedarse atrás, todas deben cumplir los objetivos propuestos (Prieto Castillo, 2005, pág. 24).

El trabajo en equipo, es importante para lograr el aprendizaje. Porque cada integrante del equipo de Regalado asume su responsabilidad desde la planificación, el desarrollo y la evaluación de la actividad de aprendizaje. Para intercambiar experiencias de aprendizaje, lograr los objetivos y metas, propuestas en las reuniones de coordinación (Cardona \& Wilkinson, 2006, pág. 2). 
Así mismo el equipo de Regalado, entiende al aprendizaje desde la perspectiva etnoeducativa. Como la participación de sus compañeras en el equipo, proponiendo objetivos claros y precisos. Para buscar el nivel de logro de las competencias, asignando tareas y funciones a las integrantes; así el conductismo, en este sentido no tienen cabida ni presencia (Freije Graña, 2009, pág. 1).

El concepto de interaprendizaje en equipo establecido por las integrantes es asignar tareas y funciones a las integrantes según sus capacidades. Como designar a la coordinadora de turno, las que van a escribir en el papelote y pegar las imágenes, las expositoras y las de soporte, las que darán respuestas a las preguntas de los otros equipos. Para lograr mayor conocimiento y mejores resultados. El interaprendizaje que el equipo de Regalado pone en práctica es la propuesta del CNEB, la corriente socio-constructivista de Vigotsky. Ellas aprenden desde su propia experiencia (zona real), se apoyan entre ellas y el apoyo del docente del área (zona próxima), para llegar a la zona potencial. Para el logro del aprendizaje propuesto por el equipo (Carrera \& Mazzarella, 2001, págs. 42-44) (Educación, marzo 2017, pág. 29).

El desarrollo de la sesión de aprendizaje en el equipo de Regalado. Como parte de la organización en equipo, siguen diferentes momentos como el inicio, proceso y salida, acompañada con los respectivos procesos pedagógicos: motivación, saberes previos, situación significativa, el nuevo conocimiento, conflicto cognitivo, trabajo en equipo, exposición y evaluación. (Pacheco Peña \& Porras Cosme, 2014, págs. 77-83).

El diseño del proyecto establecido por el equipo de Regalado fue etnoeducativo. Como parte de investigación cualitativa, que tiene un carácter holístico. Para conocer las experiencias de interaprendizaje del logro de sus competencias, desde una visión general y luego los elementos particulares de mayor significación, siguiendo el proceso inductivo (Hernández-Sapieri \& Mendoza Torres, 2018, págs. 538-539) (Martínez Miguélez, 2004, pág. 181)

Se registran los siguientes datos, codificación y análisis de datos. Se elaboró un instrumento de recojo de información con 12 preguntas, se enviaron a 5 estudiantes del equipo de Regalado, vía whatsapp web. Para obtener datos, que servirán como información, para analizarlos y dar respuesta a la pregunta de la investigación, y así generar un nuevo conocimiento. (Valderrama Mendoza, 2018, pág. 268)

La metodología etnoeducativa ha seguido pasos constructivos que van emergido de la investigación. Como el desarrollo de la observación directa y el empleo de una encuesta. Para recolectar datos, luego ser interpretados, de la forma objetiva, luego se describe las experiencias, para construir la teoría. Se empleó la ruta de la exploración.La validez de la investigación se ha trabajado desde la observación y experiencia directa con el equipo de Regalado. A través de un cuestionario de Google Drive, que ellas han respondido y luego las enviadas. Para conocer las formas de planificar, organizar, direccionar y evaluar el trabajo en equipo en el interaprendizaje y conocer el logro de sus objetivos propuestos establecidas en sus reuniones. La confiabilidad interna. Se ha realizado tomado en cuenta a los docentes de la Institución Educativa Ángela Moreno de Gálvez de Tarma, que trabajan en las aulas formando equipos de interaprendizaje. Para comparar las experiencias diversas que van experimentando los docentes y las estudiantes, especialmente el equipo de Regalado en sus diversas sesiones de aprendizaje.

Los investigadores endógenos, se conformaron con un equipo de trabajo, integrados por el Dr. Waldemar Cerrón Rojas y Dr. Jorge Yangali Vargas.

\section{Conclusiones}

El trabajo en equipo contribuye en el logro de los aprendizajes, porque cada estudiante se involucra desde la planificación hasta la evaluación, para lograr sus objetivos y metas propuestas.

Con la asignación de tareas, funciones y el cumplimiento de las normas de convivencia se logra el desarrollo de las competencias.

El interaprendizaje contribuye en el equipo de trabajo el intercambio de ideas, de responsabilidades en el proceso de aprendizaje en el aula y fuera de ella. 


\section{Referencias Bibliográficas}

Aguilar, A. (2001). Comunicación organizacional interna: proceso, disciplina y técnica. España: Netbiblo.S.L.

Cardona, P., \& Wilkinson, h. (2006). Trabajo en equipo. Comunicación organizacional interna: proceso, disciplina y técnica, 2.

Carrera, B., \& Mazzarella, C. (2001). Vygostky: enfoque sociocultural. Educare, 41 - 44.

Educación, M. d. (marzo 2017). El Currículo Nacional de la Educación Básica. Lima - Perú: Ministerio de Educación.

Freije Graña, I. (2009). Revista digital para profesionales de la enseñanza. Temas para la educación, 1

Hernández-Sapieri, R., \& Mendoza Torres, C. P. (2018). Metodología de la investigación: las rutas cuantitativ, cualittiva y mixta. México: Mc Graw Hill Interamericana Editores, S.A deC.V.

Martínez Miguélez, M. (2004). Ciencia y arte la metodología cualitativa. México: Trillas

Olivares Olivares, S. L., Torres, E. A., Avila Palet, J. E., Turrubiates Corollaa, M. L., López Cabrera, M. V., \& Valdez-García, J. E. (2018). Valor percibido de una experiencia de inmersión educativa para el desarrollo de competencias trasversales: Semana I. Educación Médica, 94

Pacheco Peña, E. M., \& Porras Cosme, S. (2014). Los momentos de la sesión a través de las rutas de aprendizaje. Propuesta de trabajo para los alumnos de las carreras de educación secundaria de la UNDAC. Horizonte de la Ciencia, 77 - 83.

Prieto Castillo, D. (2005). El interaprendizaje como clave de la educomunicación. Mediaciones, 24

Valderrama Mendoza, S. (2018). Pasos para la elaborar proyectos de investigación científica. Lima: San Marcos. 